\title{
CENTROS DE INNOVACIÓN, DESARROLLO TECNOLÓGICO Y EMPRENDIMIENTO: CASO UNIVERSIDAD DE PANAMÁ Centers innovation, technological development and entrepreneurship: Case of the Universidad de Panamá
}

\author{
Omar O. López Sinisterra ${ }^{1}$ \\ Universidad de Panamá
}

Recibido: 4-8-2015

Aceptado: 19-12-2015

\section{Resumen}

En el presente artículo se brinda un panorama general de los Centros de Innovación, Desarrollo Tecnológico y Emprendimiento, describiéndose el caso de la Universidad de Panamá (CIDETE), con sus centros en las zonas provinciales de Colón, Panamá Oeste, Bocas del Toro, Veraguas, Coclé, Herrera, Los Santos, y en el Distrito de San Miguelito, provincia de Panamá. El objetivo del estudio es presentar una recopilación bibliográfica de estos centros a través de la investigación documental basado en revistas especializadas, base de datos, google académico, catálogos on-line y la experiencia de centros similares a nivel de Latinoamérica, como el caso de Perú, Chile y Colombia. Se concluye que estos centros tienen en común líneas de investigación como la incubación de proyectos, formación cualificada de sus estudiantes en emprendimiento hacia negocios en diversas áreas, actividades de investigación y académicas enfocado a la comunidad estudiantil y público en general.

Palabras Clave: Innovación, tecnología, emprendimiento, centros tecnológicos.

\section{Abstract}

In this article we provide a general overview of the Centers of Innovation, Technological Development and Entrepreneurship; the Universidad de Panamá Case (CIDETE) is being described, along with its centers in the provincial areas of Colón, Panamá Oeste, Bocas del Toro, Veraguas , Coclé, Herrera, Los Santos, and in the District of San Miguelito, province of Panama. The aim of the study is to present a bibliographic compilation of these centers through documentary research based on journals, databases, google scholar, online catalogs and the experience in similar centers at the extent of Latin America, as in the case of Peru, Chile and Colombia. It is concluded that these centers have in common research lines as the incubation of projects, qualified student training in entrepreneurship to do business in various fields, research and academic activities focused on the school community and general public.

Keywords: Innovation, Technology, Entrepreneurship, Technological Centers.

1 Licenciado en Derecho y Ciencia Política, Director de los Centros de Tecnología, Innovación y Emprendimiento de la Universidad de Panamá. 


\section{INTRODUCCIÓN}

El fin de toda universidad es buscar estrategias innovadoras que ayuden al crecimiento de la región y del país, aunando esfuerzos académicos, investigativos y tecnológicos a través de la creación de centros de innovación y desarrollo tecnológico que coadyuven en favor de su región y del país en general. Desde esta perspectiva y cumpliendo con los objetivos estratégicos planteados y de acuerdo a su visión, la Universidad de Panamá ha creado los Centros de Innovación, Desarrollo Tecnológico y Emprendimiento (CIDETE) para devolver al país lo que este le ha otorgado desde su creación en 1935. Según lo aprobado en Consejo Administrativo del 9 de febrero del 2012 por la universidad y teniendo como fines el considerar erradicar la pobreza, impulsar el motor regional a través de la incubación de empresas, desplegar la capacidades de la población económicamente activa en las TIC's. Estimular la creatividad, desarrollando la investigación utilizando los últimos avances tecnológicos generando la creación de patentes en las provincias. Disminuir la deserción que afronta la población escolar, incorporándolo al mercado laboral en calidad de mano de obra calificada. (Dirección General de Planificación y Evaluación Universitaria Departamento de Planificación Administrativa, 2012 p.11). Lo mencionado es posible debido a que se cuenta con los tres elementos necesarios de sistemas de innovación: "infraestructura científica, personal de investigación altamente calificado y vinculación con las empresas productivas" (López, 2013, p. 42).

Ondategui $(2004$, p. 3) refiere que la creación de estos centros es una estrategia adecuada, cuyas actividades generan, adaptan y difunden conocimiento, tecnología y servicios avanzados orientados a la producción de ciencia, innovación y tecnología, introduciendo cambios en cinco aspectos: el científico, donde se realiza la producción de conocimiento; el tecnológico, donde se desarrollan las tecnologías; el entorno productivo, capaz de generar valor añadido y el financiero, que proporciona recursos a los tres aspectos antes mencionados. Vañó y Garcia (2012, p. 4) resaltan que la investigación, desarrollo tecnológico y la innovación que se realiza en un país, son factores críticos para determinar el crecimiento económico, el nivel de bienestar y su competitividad internacional. La Universidad Nacional de Panamá consideró estos aspectos y fundó la estructuración de los CIDETE, concebidos para cumplir con dos ejes principales: El vertical y el eje horizontal.

La función del eje vertical es potenciar y asesorar a los diferentes actores socio-productivos del país para contribuir de manera efectiva con el desarrollo, y concienciar mediante la educación y ejecución de programas a los estudiantes, profesores y administrativos en las zonas provinciales del país. Además, cabe resaltar que su misión consiste en promover y generar capacidades, emprendimientos, asesorías a los diferentes actores socio-productivos de Panamá, para contribuir con el desarrollo del país, así como el fortalecimiento de productores, empresarios, emprendedores e instituciones, en sus diferentes ámbitos de producción. Se trata de potenciar la gestión de la producción en las provincias, la generación de un clima de oportunidades para la competitividad, tanto como propiciar la investigación aplicada con base en el conocimiento y la tecnología y la promoción de esfuerzos concentrados a nivel institucional y empresarial en beneficio del país. En la figura 1 se muestra el local del CIDETE de Panamá Oeste.

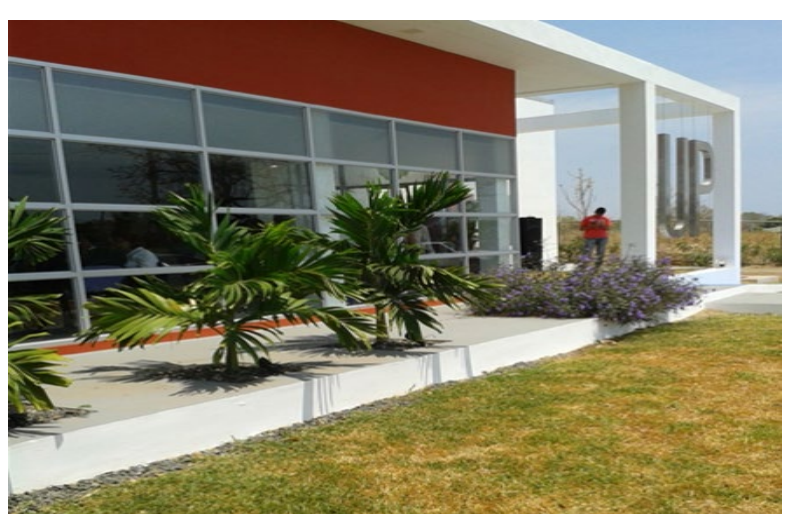

Figura 1. Fachada del CIDETE de Panamá Oeste. Fuente: Elaboración propia

El eje transversal lleva a los CIDETE al apoyo y realización de diferentes proyectos de investigación aplicada, tendientes a solucionar la problemática y necesidades a nivel nacional y que actualmente constituyen prioridades de la gestión 
presidencial. López (2013) afirma que uno de los retos de la universidad es atender la problemática nacional y regional en materia de temas económicos y sociales. Este objetivo se enmarca en la misión de la Universidad de Panamá: contribuir con el Desarrollo Nacional. Así se pretende impulsar la dinámica de desarrollo regional en base a la incubación de empresas y la creatividad en la población, a través de la investigación aplicada y la generación del impacto deseado. Según Arraut, Amar y Duque (2009), un centro de emprendimiento universitario es el que gira en torno al fomento de la cultura emprendedora, tanto para la comunidad académica de la universidad, como para la región. Baena, Jairo y Montoya (2006) enfatizan que los centros de desarrollo tecnológicos o productivos sirven para potenciar los conocimientos para nuevos desarrollos, al tener capacidad tecnológica para mejorar procesos e identificar las necesidades y buscar soluciones a estas y al estar relacionados con los diversos sectores de la región y del país. Asimismo, los CIDETE en materia de comunicación y educación, pretenden contribuir con la población nacional, sobre todo a nivel productivo, generando capacitación en emprendimiento y asesorías a las empresas de los sectores regionales, los cuales cuentan con tecnologías modernas dirigidas a mejorar el clima organizacional de las empresas, favorecer el proceso enseñanza aprendizaje y brindar servicio al sector salud de acuerdo a tecnologías de simulación clínica en áreas sensibles de la medicina a nivel nacional.

\section{CENTROS DE INNOVACIÓN, DESARROLLO TECNOLÓGICO Y EMPRENDIMIENTO}

Considerando el crecimiento que tiene la economía, sobre todo en países en vías de desarrollo, adquiere mayor envergadura la creación o cimentación de centros tecnológicos, de innovación o aquellos conformados por desarrollo tecnológico, innovación y emprendimiento. Según Fernández (2010), estos centros son entidades que se ubican entre el mundo empresarial, el académico o científico. Para la OCDE (1997), citada por San- ta María (2001), son un conjunto de actividades conducentes a nuevos procesos y/o productos (innovación radical) o mejoras tecnológicas significativas (innovación incremental). Por tanto, estos conceptos se pueden extrapolar a los CIDETE, que están coordinados por la Dirección General de Innovación, Desarrollo Tecnológico y Emprendimiento. Se encargan de dirigir y gestionar relaciones con otras instituciones a nivel nacional e internacional, la consecución de fuentes de financiamiento, programas y proyectos, capacitación a los diferentes actores socio-productivos. Debido a que estos centros deben tener un acercamiento con la región e involucrar a sus autores, la Universidad Nacional de Panamá ha ubicado a estos centros en el distrito de San Miguelito, las provincias de Panamá Oeste, Herrera, Colón, Los Santos, Veraguas, Coclé y Bocas del Toro, en los Centros Regionales Universitarios.

Los CIDETE han sido creados para proveer a la comunidad nacional de un área disponible para desarrollar creatividad en base a ideas que puedan desarrollarse en productos y servicios de interés general, brindar un apoyo en base a la tecnología de la información y la comunicación a los empresarios, emprendedores sectoriales y al país. Pese a que en España son definidos como centros de Investigación y Tecnología en España, esta definición está dentro de lo estipulado por CIDETE: "son organizaciones que contribuyen al desarrollo económico y social del país, su estrategia es apoyar e impulsar todos los procesos de innovación y desarrollo tecnológico, a fin de que el entorno empresarial alcance competitividad industrial" (Santa María, 2001, p. 35). Esto con el fin de mejorar y acrecentar el conocimiento de los actores socio-productivos en el campo digital; promover y generar talentos nacionales que participen en los espacios de los centros, en los cuales pueden realizar investigaciones bajo la asesoría de los especialistas universitarios; gestionar proyectos de interés nacional e internacional dirigidos a solucionar problemas y necesidades locales y, a su vez, desarrollar capacidades científicas y tecnológicas. CreceMype (s.f) refiere que estos centros "son instituciones que actúan como socios tecnológicos, vinculando al conocimiento y contribuyen 
a incrementar la producción, el empleo de calidad y la competitividad". Por su parte, la UC3M (s.f) refiere que estos centros son instituciones sin ánimo de lucro y se centran en el desarrollo de proyectos de innovación y desarrollo $(\mathrm{I}+\mathrm{D})$. Casado y Serrano (2008) enfatiza que estos centros contribuyen a que las comunidades científicas compartan sus experiencias y se nutran de las investigaciones realizadas, así como permiten el desarrollo de la investigación en diversas áreas del conocimiento en el país en todos los campos de la sociedad. En esta misma línea, Gracia y Segura (2003) aseveran que los centros tecnológicos actúan como puente entre las universidades y el desarrollo de la investigación básica, siendo un valor agregado como aliados en aspectos estratégicos de la universidad-empresa; son un factor del sistema: ciencia-tecnología-sociedad.

La experiencia en Perú relacionada a estos centros como lo menciona Ponce (abril 13, 2013) es que están inmersos dentro del ámbito académico en diversas universidades e institutos, teniendo como finalidad el unir conocimiento, innovación, tecnología y emprendimiento social, así como el desarrollo de proyectos tecnológicos a través de la incubación. Destacan el Centro de Innovación y Emprendimiento (CIE) de la Universidad Nacional Mayor de San Marcos, que tiene como fin formar empresas y empresarios, a través de la incubación de empresas tecnológicas, iniciando sus actividades el 2012. Desde su creación ha formado 2950 emprendedores, 351 empresas, que han obtenido financiamiento del Fondo para Innovación, Ciencia y Tecnología de CONCYTEC y realizado 1966 planes de negocios.

El Centro de Innovación y Desarrollo Emprendedor (CIDE) de la Pontifica Universidad Católica del Perú, el cual imparte diplomados y cursos para emprendedores, así como brinda asesoría para creación de empresas, gestionar la sostenibilidad de empresas, apoyo en consultoría para proyectos de innovación e incubación. Asimismo Emprende UP (2015) menciona que el Centro de Emprendimiento e Innovación UP (Emprende UP) de la Universidad del Pacifico, tiene como objetivos el promover una cultura emprendedora en la comunidad universitaria para el desarrollo socioeconómico del país, así como la investiga- ción, la capacitación y la consultoría en actitudes y actividades emprendedoras para articularse con el mercado empresarial e inversionistas; impulsando que los estudiantes cimenten su cultura emprendedora hacia la de empresario a través de un proyecto innovador. Según el Centro de Emprendimiento USIL, de la Universidad San Ignacio de Loyola, tiene como objetivo lograr que la totalidad de los estudiantes al concluir su carrera profesional hayan desarrollado paralelamente un centro empresarial, para ello se apoya en la incubadora Start-Up USIL.

Chile, es otro de los países de Latinoamérica que también ha desarrollado centros de Innovación, Desarrollo Tecnológico y emprendimiento, uno de ellos es el Centro de Innovación y Transferencia Tecnológica de la Universidad de Chile, que a través de una plataforma, busca promover gestionar, negociar y comercializar proyectos de innovación y desarrollo con vinculación de instituciones públicas y privadas a nivel nacional $\mathrm{e}$ internacional, brindando servicios de transferencia tecnológica, emprendimiento e incubación de negocios, difusión tecnológica, tracking de proyectos y formación y desarrollo tecnológico (Innovo, 2015). El centro de Innovación UC Anacleto Angelini de la Pontificia Universidad Católica de Chile, tiene como objetivo fomentar la cultura del emprendimiento e innovación para generar iniciativas en que participen empresas, emprendedores e investigadores con proyectos de alto impacto social y económico, dentro de sus actividades organizan los Concursos Nacionales de Emprendimiento Universitario "Jump Chile", científico-tecnológico "BRAIN UC" y el de innovación social "CoLb Alto Impacto" (Centro de Innovación UC, 2015). La Universidad Católica del Norte dentro de los centros de innovación, desarrollo tecnológico y emprendimiento cuenta con el Centro de Investigación en gestión de tecnologías para la empresa (CIGTE), tiene como fin desarrollar investigación aplicada en áreas de la gestión y de las tecnológicas para el mejoramiento de las empresa de la zona norte de Chile, con innovación empresarial, gestión estratégica y gobierno de las tecnologías y desarrollo tecnológico, otro de sus centros es el Centro de Emprendimiento y de la Pyme (CEMP) siendo su fin el de educación e investigación aplicadas a las áreas de 
emprendimiento y desarrollo de la pequeńa y mediana empresa, vinculando universidad-empresa (Universidad Católica del Norte, 2015).

En Colombia, la Universidad de Medellín cuenta con un centro de Innovación y Desarrollo empresarial, la cual tiene como objetivos, la transferencia de conocimiento, emprendimiento de base tecnológica y de innovación, gestionar la propiedad intelectual, transferencia de conocimiento e innovación y tecnología, vinculando Universidad-empresa-estado y sociedad (Centro de Innovación y Desarrollo Empresarial, 2015). La Universidad Militar Nueva Granada, desde la perspectiva de desarrollar una cultura empresarial en sus estamentos, para vincular universidad y el sector empresarial, mediante Resolución 3726 del 2013, ha impulsado la creación de un centro de Innovación y Emprendimiento Neogranadino, que tiene como objetivos realizar transferencia tecnológica, proyectos de innovación, StartUp, proyectos de Incubación, emprendimiento dirigida a todos los miembros de su comunidad académica y el entorno empresarial (Unidad de Emprendimiento e Innovación, 2015).

\section{EJES ESTRUCTURALES DE LOS CIDETE}

De acuerdo con las funciones que se realiza en los Centros de Innovación, Desarrollo Tecnológico y Emprendimiento, se estructuraron dos ejes que contienen diversos procesos. La figura 2 presenta los ejes y sus funciones.

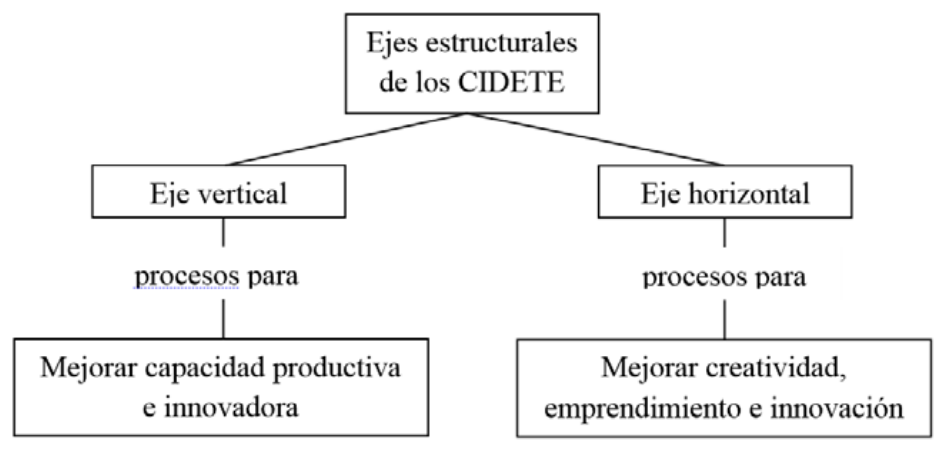

Figura 2. Funciones de los ejes estructurales de los CIDETE.

Fuente: Elaboración propia
En el eje vertical se introducen aquellos dirigidos a mejorar la capacidad productiva, emprendedora e innovadora de los diferentes actores socio-productivos del área a nivel de provincias, mediante la orientación adecuada y el soporte tecnológico que se les brinde con la tecnología existente en los centros. Así, se capacita a los actores socio-productivos de la geografía nacional utilizando las herramientas tecnológicas para que incida positivamente en sus capacidades creativas, innovadoras, emprendedoras y productivas, impactando de esta manera en el desarrollo del país. Naturalmente, esto permite una amalgama resultante de la convergencia entre el sector productivo, de servicios y el sector académico universitario, lo cual también lo enfatiza la Universidad de la Salle, al mencionar que desde el interior de la universidad, las políticas públicas de ciencia, tecnología e innovación contribuyen a la solución de problemas sociales.

En el eje horizontal se incluyen los procesos diversificados tendientes a mejorar la creatividad, el emprendimiento, y la innovación en el área de la enseñanza y el aprendizaje universitario en materia de redes, tecnología educativa, generación de nuevas técnicas de enseñanza y el uso de los simuladores clínicos en las facultades de Medicina y Enfermería.

Los CIDETE son unidades enquistadas en los Centros Regionales Universitarios y dependen de ellos administrativamente, mientras que en su parte funcional y razón de ser dependen de la Dirección General de Innovación, Desarrollo Tecnológico y Emprendimiento, con la que desarrollan programas, proyectos, capacitaciones, asesorías, convenios y fuentes de financiamiento.

\section{INFRAESTRUCTURA Y ÁMBITO GEOGRÁFICO DE LOS CIDETE}

Lozano (2012) considera pertinente afianzar la vinculación universidad-empresa en áreas prioritarias para la investigación ligadas a distintos ámbitos de interés regional y que respondan a las problemáticas que viven las comunidades y regiones. Desde esta perspectiva, los CIDETE han sido desarrollados en una infraestructura acorde a 
las necesidades de poblaciones vulnerables, respetando el ámbito geográfico y siguiendo un modelo de gestión idóneo para los mismos.

Como menciona Baena et al. (2006), el papel de los centros de desarrollo es permitir el bienestar de las comunidades para lograr un desarrollo económico y social de las regiones. Por intermedio de ellos se logran procesos de innovación que mejoran la productividad y la competitividad empresarial. Teniendo en cuenta estos aspectos, los CIDETE han sido situados en áreas específicas de los Centros Regionales Universitarios, habiendo iniciado sus labores desde el 2014, de acuerdo a lo normado por el Art. 8 de la Ley Orgánica, los estatutos de la Universidad de Panamá y Acuerdo de Consejo Administrativo. Cada uno de los CIDETE posee en su estructura física áreas especialmente diseñadas para lograr los objetivos propuestos, contando con una infraestructura adecuada. López (2013) refiere que los laboratorios, talleres, equipos de cómputo, bibliotecas deben ser los más idóneos y adecuados para estos centros. A continuación, se detallan brevemente los mismos.

a. Vestíbulos. Es el área donde se explica a los usuarios del sistema cómo funcionan los diversos prototipos con que se cuenta en cada uno de los centros, además de los programas, proyectos y productos como desarrollo de la labor realizada. La figura 3 muestra la sala de recepción del CIDETE de Colón.

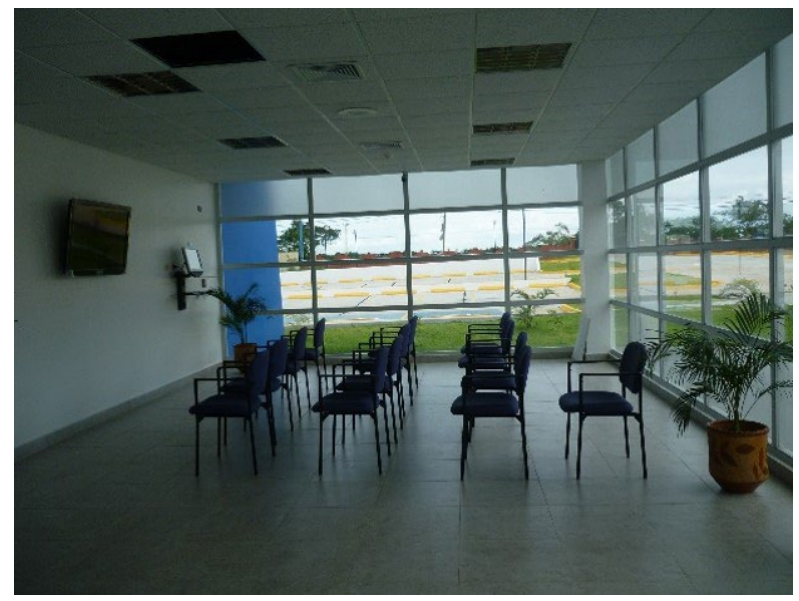

Figura 3. Sala de recepción del CIDETE de Colón. Fuente: Elaboración propia

b. Áreas de videoconferencias. Es la zona en donde se realizan conferencias a nivel nacional e internacional. Brinda servicios a todas las instituciones privadas como públicas para contribuir al desarrollo de la comunicación y participación del conocimiento. Tendrán también participación muy activa las universidades nacionales e internacionales con las cuales se han desarrollado convenios. La figura 4 muestra el ejemplo de una videoconferencia realizada en el CIDETE de Bocas del Toro, mientras que en la figura 5 se observa al público asistente a una videoconferencia desarrollada en este mismo centro.

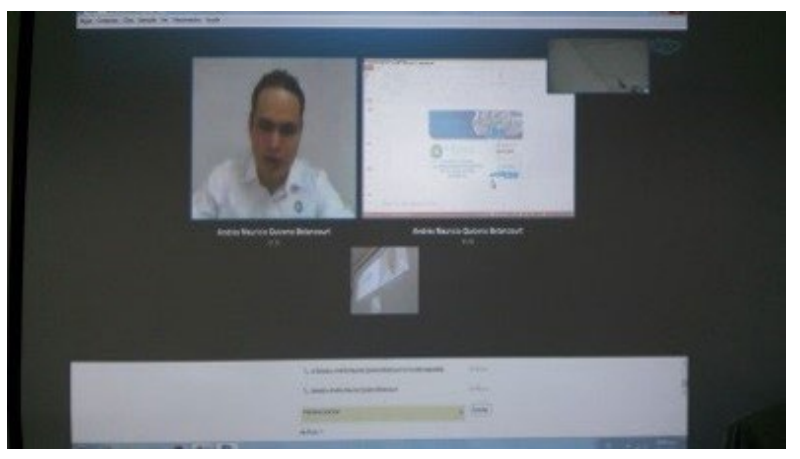

Figura 4. Ejemplo de videoconferencia realizada en el CIDETE de Bocas del Toro.

Fuente: Elaboración propia

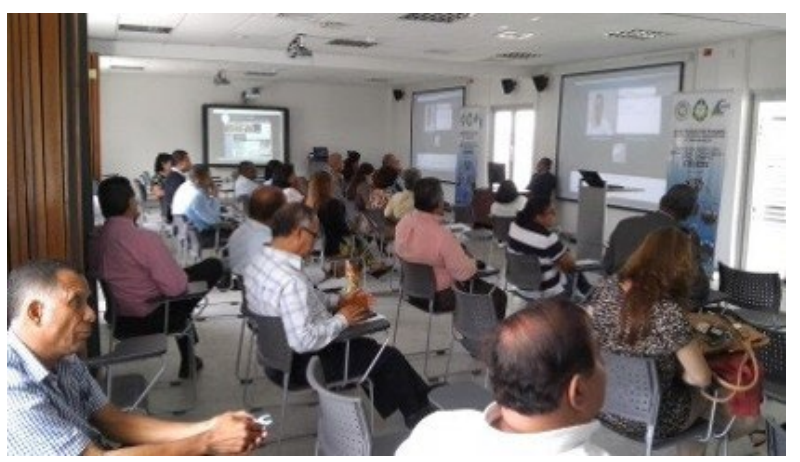

Figura 5. Público asistente a una videoconferencia realiazda en el CIDETE de Bocas del Toro.

Fuente: Elaboración propia

c. Salones inteligentes. Constituyen áreas específicas para la transmisión del conocimiento en diversas especialidades. La innovación, el desarrollo tecnológico y el emprendimiento son los temas primarios a desarrollar en los mismos. En estos centros converge el conocimiento y concitan la participación conjunta de profesionales del sector público, privado y 
profesores especialistas universitarios. En la figura 6 se muestra una sesión de trabajo desarrollada en el Salón Inteligente del CIDETE de Panamá Oeste.

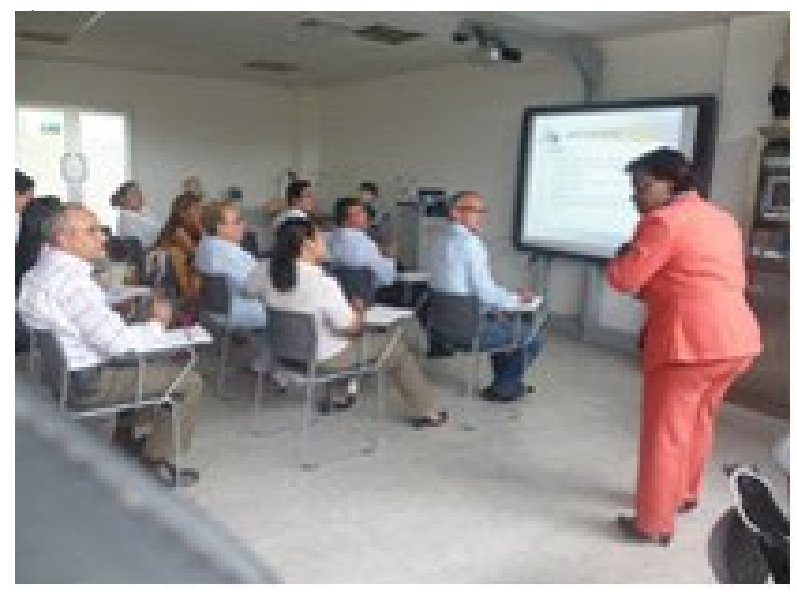

Figura 6. Desarrollo de una sesión de trabajo en el Salón Inteligente del CIDETE de Panamá Oeste Fuente: Elaboración propia

d. Laboratorios de creatividad. Son áreas para la generación del conocimiento en base a ideas de los diferentes actores socio-productivos que interactúen en ellos. Es el área de capacitación de los profesores y en donde se realizan prácticas para generar ideas que podrán ser el foco de nuevas investigaciones y creaciones de impacto en la comunidad. Los programas disponibles en los CIDETE son utilizados para incentivar y servir de instrumentación para el fin propuesto.

e. Área de Simulación Clínica. Destinada a ofrecer servicios en Ciencias de la Salud en diferentes especialidades médicas. Están al servicio de los Complejos de Salud tanto públicos como privados, incluyendo el sistema hospitalario. Este sistema permite realizar prácticas en diversos procedimientos médicos sin que el usuario esté expuesto a los riesgos propios de la atención a seres humanos. Permite también que los médicos y enfermeras de las instituciones de salud realicen procedimientos quirúrgicos de innovación controlando su ejecución y resultados sin incluir al paciente. La figura 7 muestra la estructura del Área de Simulación Clínica en el CIDETE de Colón.

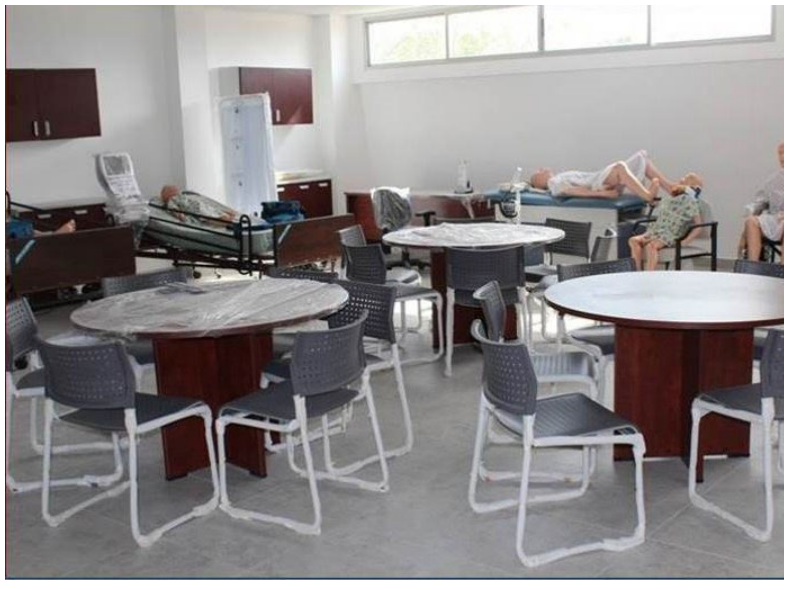

Figura 7. Área de Simulación Clínica, CIDETE de Colón.

Fuente: Elaboración propia

f. Zona Abierta o Espacio Abierto. Es un área destinada a la presentación de los proyectos diseñados, en desarrollo y terminados, generados por los diferentes actores socio-productivos, incluidos profesores, estudiantes y administrativos de la universidad. Naturalmente, esto permite la conjugación de la universidad con los miembros de la sociedad y comunidad en general.

\section{MÉTODO}

Esta es una investigación de tipo bibliográfica, que exploró la literatura correspondiente a experiencias e investigaciones realizadas por los Centros de Innovación y Emprendimiento en diversos países y universidades. La selección del material bibliográfico y documental se hizo analizando las experiencias, conocimientos y trayectoria de los CIDETE de Latinoamérica, según los siguientes criterios:

a. Experiencia (años de funcionamiento).

b. Tipo de Centro (empresarial, innovación, desarrollo tecnológico, emprendimiento).

c. Grados de avance en su competencia.

d. Similitud con la labor a realizar por los CIDETE.

e. Reconocimiento de labor realizada y sus aportaciones.

Después de haber seleccionado los Centros de Innovación en Chile, Perú y Colombia, se progra- 
maron visitas in situ para conocer de manera precisa el proceso de ejecución de la labor desplegada por los mismos en esta materia. La información obtenida también fue utilizada para la organización de los CIDETE y para realizar la estructura de funcionamiento y se describa en el presente artículo.

\section{IMPACTO DE LOS CIDETE EN LA EXPERIENCIA DE LA UNIVERSIDAD DE PANAMÁ}

$\mathrm{Al}$ ser un estudio documental solo se describe el impacto que ha tenido los CIDETE y su equipo humano, más no se puede hacer referencia a resultados por no tener datos cuantificados, en el caso de la Universidad de Panamá, entre sus actividades han realizado diferentes proyectos destinados a satisfacer necesidades primigenias del país. Entre ellos: el "Tratamiento de los Desechos Sólidos", "Tratamiento de los Desechos Hospitalarios", "Tratamiento del Agua", etcétera. También se han realizado acciones de capacitación en emprendimiento a los actores socio-productivos que han incursionado en esa área. Con instituciones como SENACYT se está realizando convenios; primeramente se utilizarán "Space Makers" en los salones de creatividad en los CIDETE del Centro Regional de Veraguas, el de Herrera y el de Panamá Oeste. Se ha realizado una alianza con la Autoridad de la Pequeña y Mediana Empresa (AMPYME) para fortalecer e impactar el Desarrollo Nacional. Finalmente, se ha previsto realizar una simbiosis sin precedentes con el Estado, la Empresa Privada y la Comunidad en general. La realización de la FERIA y del FORO de los CIDETE se ha constituido en la piedra angular para el desarrollo de esta estrategia. Estas experiencias innovadoras permiten que la universidad cumpla su rol de mitigar problemas de su entorno, devolviendo de alguna manera al Estado los beneficios que este le ha proporcionado; a su vez, honra la confianza que la comunidad educativa puso en ella para formar profesionales que egresen y se inserten fácilmente en el campo laboral según las necesidades del ámbito productivo.

Además se ha formulado proyectos en diferentes áreas sensibles de la vida nacional respondiendo a necesidades urgentes y a eventos significativos del acontecer nacional e internacional, algunos de ellos son la presentación del informe del PNUD; Conferencia de AUPSA (Seguridad Alimentaria), Capacitación del personal de CIDETE, Taller sobre "De la Idea al Negocio", Seminario Internacional de Álgebra no Conmutativa, Foro "Riesgo, Seguridad Hídrica y Cambio Climático en el Distrito de Penonomé", Seminario de Capacitación en Simuladores Clínicos, Jornadas de Vídeo Conferencias de la Caja del Seguro Social, VI Encuentro de Software Libre, Herramientas para Desarrolladores, Talleres de Creatividad, Tecnologías de la Comunicación en Educación, Seminario On Line con IPN México, Presentación del Proyecto de los Vertederos, Proyecto "el Arte de la Biología Molecular en Panamá Oeste” entre otros.

\section{CONCLUSIONES:}

De acuerdo al análisis realizado entre los CIDETE de la Universidad de Panamá y los de Chile, Perú y Colombia, se han encontrado líneas de investigación en común como son, la incubación de proyectos, formación cualificada de sus estudiantes en emprendimiento hacia negocios en diversas áreas, actividades de investigación y académicas enfocado a la comunidad estudiantil y público en general, desarrollo tecnológico, impulsar la transferencia tecnológica al mercado empresarial, así como gestionar y generar conocimiento empresarial en la formación de sus estudiantes antes de que egresen a través de la creación de centros por medio de los StarUp o la incubación de empresas.

Asimismo los cambios en la economía, en el mundo empresarial y en la sociedad en general han contribuido a que las universidades a nivel de Latinoamérica busquen alternativas para convertirse en un engranaje con la sociedad, generando innovación, tecnología y emprendimiento a través de los CIDETE, como lo demuestra la experiencia en países como Perú, Chile y Colombia, en donde converge una similitud al haber desarrollo en estos centros para generar creaciones, innovación a través de la incubación de empresas, gestionar patentes, capacitar a la comunidad en general, impulsar la relación empresa-universi- 
dad, así como la estimulación de la creatividad en los diferentes actores socio-productivos, es en definitiva una fortaleza y una ganancia para impactar en el desarrollo socio-económico de nuestros países, los cuales poseen características propias y unidireccionales en cuanto a la temática en la que han desarrollado, incluyendo la infraestructura, los equipos, los laboratorios entre otros aspectos.

Si bien los CIDETE son una alternativa para paliar las carencias que se tiene a nivel de políticas públicas y un mayor aporte del estado para revertir problemática ya conocidas, no se puede dejar de mencionar que el apoyo que está brindando las universidades con el aporte de conocimiento, emprendimiento, innovación y transferencia tecnológica, a través de estos centros es muy valiosa, porque permite que personal cualificado con experiencia y experticia en el tema brinde a la sociedad y a la nueva comunidad universitaria los elementos necesarios para que se comprometan con este nuevo enfoque de unir innovación, desarrollo y tecnología.

Los CIDETE requieren realizar transferencia del conocimiento a través de la tecnología de la información y la comunicación, generar la creatividad en los diferentes actores y cooperar con la transferencia de la tecnología que existe a disposición de estos mismos. Asimismo, brindan la oportunidad de tejer una red de relaciones con los diversos centros en países hermanos y continuar una vinculación investigativa, académica y productiva de innovación, no solo en el apoyo a los estudiantes sino a la comunidad y la sociedad, al estar en condiciones de aplicar tecnología de punta, intercambiar recursos, conocimientos y experiencias.

Se recomienda que para futuras investigaciones relacionadas a los CIDETE sería importante verificar si estos se han desarrollado aunando esfuerzos con la vinculación con otros centros que existen en Latinoamérica y Europa que comparten objetivos en común y problemática a fin o si continúan impulsándose de manera aislada en cada país, como se está realizando en la actualidad y cuáles son las razones de no hacerlo colaborativamente.

Sería relevante hacer una medición de estos centros, tanto en inversión como en comercializa- ción, registrando de manera cuantificable, a través de una base de datos los resultados obtenidos en cada uno de los centros y unidades por año pertenecientes a los CIDETE.

\section{REFERENCIAS BIBLIOGRÁFICAS}

Arraut, L., Amar, P. y Duque, J. (2009). Hacia un modelo de emprendimiento de innovación abierta socialmente responsable: Caso Universidad Tecnológica de Bolívar en Colombia. Revista TEC Empresarial. 3 (1-2) 33-44.

Casado, D. y Serrano, R. (2008). Productividad del Centro de Investigación y Desarrollo Tecnológico Dr. Mario Szczurek. Revista de Investigación, 65, 33-55.

Centro de Innovación UC (2015) Centro de Innovación UC Anacleto Angelini de la Pontificia Universidad Católica de Chile. Recuperado de: http://centrodeinnovacion.uc.cl/ emprendimiento/

Centro de Innovación y Desarrollo Empresarial (2015) Universidad de Medellín. Recuperado de: http://www.udem. edu.co/index.php/lo-nuevo-en-la-vicerrectoria/informacion-del-centro-investigaciones

CreceMype (s/f) La web del empresario MYPE. Ministerio de la Producción. Recuperado de: http://www.crecemype. pe/portal/index.php/servicio-de-desarrollo-empresarial/ red-cities

CIE (2015) Centro de Innovación y Emprendimiento. Universidad Nacional Mayor de San Marcos. Recuperado de: http://www.ciesanmarcos.com/

Dirección General de Planificación y Evaluación Universitaria Departamento de Planificación Administrativa (2012) Centros de Innovación, Desarrollo Tecnologico y Emprendimiento, de los Centros Regionales Universitarios de la Universidad de Panamá-(CIDETE). Recuperado de http:// www.up.ac.pa/ftp/2010/d_planificacion/documentos/CIDETE.pdf

Emprende UP (2015) Universidad del Pacifico. Recuperado de http://emprendeup.pe/?utm_source=WEB\%20 UP\&utm_medium=Redirect\&utm_campaign=EmprendeUP

Fernández, J. (2010). Modelo de desarrollo de centros tecnológicos industriales orientados a proyectos en entornos no intensivos en innovación. (Tesis doctoral inédita). Universidad de Oviedo: España.

Gracia, R. y Segura, I. (2003). Los centros tecnológicos y su compromiso con la competitividad, una oportunidad para el Sistema Español de Innovación. Revista Economía Industrial, 354 (6), 72-84.

Innovo (2015) Centro de Innovación y Transferencia Tecnológica. Universidad de Santiago de Chile. Recuperado de: http://www.usach.cl/innovo

López, E. (2013). Las universidades como gestoras del cambio. Revista Xihmai, 8 (15), 35-56. 
Lozano, M. (2012). Tecnología e innovación en América Latina y los desafíos para la democratización de la ciencia. Revista Ciencia, Tecnología, Sociedad. 6, 1-12.

Ondategui, J. (2004). Tecnología y Servicios en los Centros de apoyo a la Innovación en Espańa. Revista de Investigación en Gestión de la Innovación y Tecnología, 25, 1-14. Recuperado de: http://www.madrimasd.org/revista/revista25/tribuna/tribuna1.asp

Ponce, F. (abril 30, 2013) Perú: Conoce los centros de Emprendimiento e Innovación universitarios más importantes. Pulso Social. Recuperado de http://pulsosocial. com/2013/04/30/peru-conoce-los-centros-de-emprendimiento-e-innovacion-universitarios-mas-importantes/

Santa María, Ll. (2001). Centros tecnológicos, confianza e innovación tecnológica en la empresa: Un análisis económico. (Tesis doctoral inédita). Universitat Autónoma de Barcelona, Departament d'Economia de I'Empresa, España.

UC3M (s.f). Un estudio analiza la actuación de las universidades y los centros tecnológicos en la innovación empresarial. Universidad Carlos III de Madrid Recuperado de: http://portal.uc3m.es/portal/page/portal/actualidad_cientifica/noticias/estudio_innovacion

Universidad Católica del Norte (2015) Centros de investigación. Recuperado de: http://www.ucn.cl/investigacion/ centros-de-investigacion/

Unidad de Emprendimiento e Innovación (2015) Centro de Innovación y Emprendimiento Neogranadino. Universidad Militar de Nueva Granada. Recuperado de: http://www. umng.edu.co/unidad-de-emprendimiento-e-innovacion1

Universidad de la Salle (s.f). Innovación y Tecnología. Vicerrectoría de Investigación y Transferencia. Recuperado de: http://www.lasalle.edu.co/wps/wcm/connect/b98917e3b2a2-4a70-ae99-cfdc56850ddc/innovaci\%C3\%B3n+y+Tecnolog\%C3\%ADa.pdf?MOD=AJPERES

Universidad de Medellín (2015) Investigación-Centro de Innovación y Desarrollo Empresarial. Recuperado de: http:// www.udem.edu.co/index.php/lo-nuevo-en-la-vicerrectoria

Vañó, L. y Garcia, M. (2012). Estudio de las herramientas de gestión de los proyectos $\mathrm{I}+\mathrm{D}+\mathrm{I}$. Revista de investigación 3 Ciencias, 1-15. Recuperado de: http://www.3ciencias.com/ wp-content/uploads/2012/08/3.herramientas-gestion-I+D+i.pdf 